\title{
Investigation and Generation of Vacancies in Alpha Quartz by Soft X-Rays
}

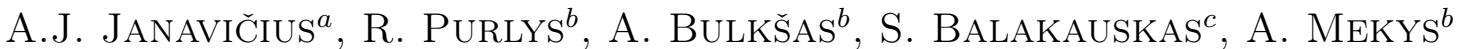 \\ AND V. ̌́ SABLINSKAS ${ }^{b}$ \\ ${ }^{a}$ Faculty of Nature, Šiauliai University, P. Višinskio 19, 76351 Šiauliai, Lithuania \\ ${ }^{b}$ Faculty of Physics, Vilnius University, Saulètekio 9, 10222 Vilnius, Lithuania \\ ${ }^{c}$ Semiconductor Physics Institute, A. Goštauto 11, 2600 Vilnius, Lithuania
}

(Received September 18, 2008; in final form October 2, 2009)

\begin{abstract}
In this paper we applied the soft-X-ray radiation for generation of point defects, vacancies, and chemical reactions in quartz $\left(\mathrm{SiO}_{2}\right)$, taking into account our earlier made similar experiments with crystal silicon and importance of quartz for applications in many fields. In this case only radiative Auger's effects with electrons and electric dipole of atoms transitions can generate metastable vacancies, point defects, and induce chemical reactions. Usually, for point defects generation doses of gamma rays are used. We measured values of the Bragg reflections of X-rays and calculated mean square deviations of atoms in crystal lattice for defining the dynamics of irradiated point defects. We accomplished infrared measurements for establishing of generated chemical reactions, and conductivity measurements were also done.
\end{abstract}

PACS numbers: 81.40.-z, 78.30.-j, 91.60.Mk

\section{Introduction}

The aim of this paper is application of soft X-rays for generation [1] and investigation [2] of point defects in quartz. The stimulated Auger effect by soft X-rays, instead irradiation with gamma rays, can be used as the alternative tool for generation of point defects [3], metastable vacancies [4-7] and induction of chemical reactions. Irradiation with soft X-rays changes conductivity $[4,6,7]$, creates resonances of charge carrier scattering effect $[4,7]$, and generates resonance phenomena in lattice with energy transitions $[6,7]$.

We will investigate the quartz as important material in many fields of applications and interesting for irradiation, because it contains many natural impurities like $\mathrm{Al}, \mathrm{Fe}$, $\mathrm{Li}, \mathrm{Na}, \mathrm{H}$ assisted by creation of interstitial point defects which induce the displacements of atoms in lattice.

Quartz can be used for radiation dosimeters, monochromators. The experiments with diffractometer require the monochromatic X-ray radiation. The $K_{\alpha}$ line can be isolated with various monochromators and among them an important role is played by quartz monochromators, which are also used as standards for tuning and testing of diffractometer.

We applied soft X-rays for investigation of dynamics of the Frenkel point defects and chemical reactions in alpha quartz crystal (the basic structural element $\mathrm{SiO}_{4}$ tetrahedron), generated by the Auger and Compton effects. The impurities, point defects, and thermal motion of atoms cause the changes of relative intensities of the Bragg re- flections $[2,5]$ before irradiation

$$
\frac{I_{i}}{I_{0}}=\exp \left(-\frac{16}{3} \pi^{2}\left\langle u_{i}^{2}\right\rangle(\sin \vartheta / \lambda)^{2}\right)
$$

and generate variations of lattice parameters and its oscillations [5]. Here $I_{0}$ is the intensity of incident beam and $I_{i}$ is the initial intensity of reflected beam. Admitting that initial mean square displacements of atoms $\left\langle u_{i}^{2}\right\rangle$ in the lattice are invariable and time dependent mean square displacements $\left\langle u(t)_{V}^{2}\right\rangle$, representing irradiated vacancies and generated chemical reactions, are independent terms, we can write $\left\langle u(t)^{2}\right\rangle=\left\langle u_{i}^{2}\right\rangle+\left\langle u(t)_{V}^{2}\right\rangle$. Substituting $\left\langle u(t)^{2}\right\rangle$ instead $\left\langle u_{i}^{2}\right\rangle$ into (1) we obtain

$$
\frac{I(t)}{I_{0}}=\exp \left(-\frac{16}{3} \pi^{2}\left(\left\langle u_{i}^{2}\right\rangle+\left\langle u(t)_{V}^{2}\right\rangle\right)(\sin \vartheta / \lambda)^{2}\right),
$$

where $I(t)$ are intensities of reflected beam, which depend on irradiation time.

Dividing (2) on (1), we found dependence of mean square displacements of atoms on time as a result of irradiation process

$$
\sqrt{\left\langle u(t)_{V}^{2}\right\rangle}=\frac{\sqrt{\ln \frac{I_{i}}{I(t)}}}{\frac{4 \pi}{\sqrt{3}} \frac{\sin \vartheta}{\lambda}} .
$$

The presented formula gives a possibility to separate the influence on the Bragg reflection of initial defects and impurities.

This presented mathematical method allows us to investigate the relaxation processes and point defects production in quartz crystal like in silicon [1]. The irradi- 
ated point defects can reconstruct the broken chemical bonds in different ways, creating the changes in quartz lattice. Metastable vacancies, trapping electrons, can exchange charge states to more probable and generate distortions in the lattice. Capture of carriers at the defects can transmit the vibration energy into the neighboring atoms, which can change its configurations [8] in the lattice. Those processes assist for migration of vacancies and atoms, change conductivity, and generate relaxations of lattice.

\section{Measurements of dynamics of the Frenkel point defects in quartz}

The samples of alpha quartz monocrystals $\left(\mathrm{SiO}_{2}\right)$ (2 mm thick), with surface parallel to the (101) plane, were polished until shining. The diffraction measurements were done using the (202) crystallographic plane, which usually is used for monochromatization. Time-dependence of the Bragg diffraction peak intensity was measured using the X-ray tube with anode voltage $U_{\mathrm{a}}=$ $20 \mathrm{kV}$ and current $20 \mathrm{~mA}$. Standard twofold diffraction angle from the (202) crystallographic plane of $\mathrm{Cu} K_{\alpha_{1}}$ line rays for alpha quartz is $2 \vartheta=55.29^{\circ}$. The time dependence of intensities of the peak for a diffraction angle $\vartheta=27.65^{\circ}$ was measured using the $K_{\alpha_{1}}$ line of characteristic X-ray radiation of $\mathrm{Cu}(\lambda=1.5405 \AA)$ at temperature $20^{\circ} \mathrm{C}$ for sample connected with the massive metallic corpus of apparatus. In this case sample temperature stayed practically constant and was equal to corpus and room temperature.

The investigations were performed with a general-purpose DPOH-3M (Russian device) diffractometer. The kinetics of diffraction peak intensity was measured during $400 \mathrm{~min}$, duration of a single measurement was $10 \mathrm{~s}$. Till $280 \mathrm{~min}$ the interval between measurements was $1 \mathrm{~min}$, later - $2 \mathrm{~min}$.

In result of the Auger effect we obtained periodic transitions of $\mathrm{Si}$ atoms into different states [4,5] and metastable vacancies generation. Thus, distribution of total intensity $I(x)$ of the Bragg profile of a reflected beam in crystal with defects can be presented [2] as sum of diffraction peak intensities for ideal crystal and intensity of diffuse scattering. The generated point defects and metastable vacancies are cause of decrease of diffraction peak intensities. Vacancies are in different charge and energy states $[8,9]$ and their transitions to more stable states with lower energies generate relaxation processes [5] and distortions in lattice. We measured time dependent oscillations intensity of the Bragg diffraction peak and it can be connected with specific periodic ultrasonic strains [10]. In Fig. 1 we have point defects and metastable vacancies production $[4,5]$ presented by oscillations of decreasing intensities.

Using diffraction peak dependence on time presented in Fig. 1 we calculated root mean square deviations (3) of lattice atoms as result of point defects produced by X-ray irradiation.

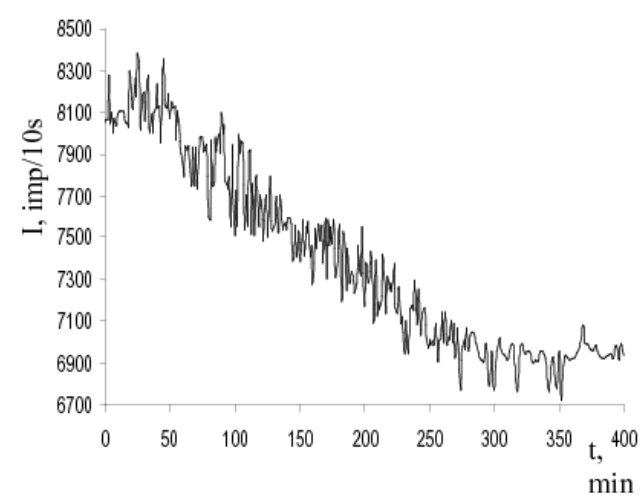

Fig. 1. Dependence of diffraction peak intensities on time $t$ (number of photons registered with $10 \mathrm{~s}$ measurement duration with 1 min interval for a diffraction angle $\vartheta=27.645^{\circ}$ ) of reflection from the (202) crystallographic plane. Experiment was done with $\mathrm{Cu}$ anode X-ray characteristic spectral line $K_{\alpha_{1}}$ of wavelength $\lambda=1.5405 \AA$ for anode voltage $U_{\mathrm{a}}=20 \mathrm{kV}$ and tube current $I_{\mathrm{a}}=20 \mathrm{~mA}$.

Results presented in Fig. 2 can be explained as cleaning of crystal from metastable point defects and metastable vacancies by diffusion till $25 \mathrm{~min}$. Later, till $300 \mathrm{~min}$, the saturation of sample by the irradiated chemical reactions and vacancies was finished. Cleaning crystal from point defects can occur only in the result of diffusion of irradiated products of chemical reactions.

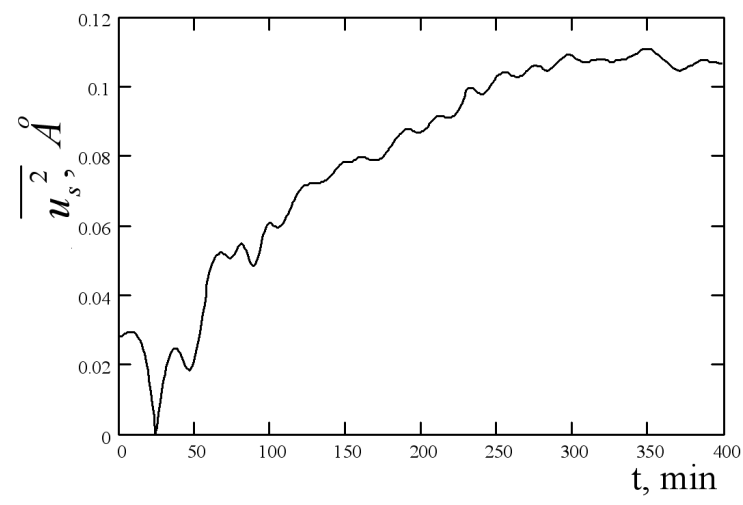

Fig. 2. Dependence of root of mean square displacements of atoms in lattice from equilibrium positions on time $t$, corresponding to measurements presented in Fig. 1.

The influence of irradiated vacancies and other point defects on the distance $d_{0}+\Delta d(t)$ between crystallographic planes of the lattice depends on time $t$ of irradiation and can be estimated from angular positions $\theta_{0}+\Delta \theta(t)$ of diffraction spectrum peaks, using the following expression for fractional change in the lattice spacing [11]: 


$$
\frac{\Delta d(t)}{d_{0}}=1-\frac{\sin \left(\theta_{0}\right)}{\sin \left(\theta_{0}+\Delta \theta(t)\right)},
$$

and the macroscopic strain $\mu$ of the irradiated sample

$$
\frac{\Delta d(t)}{d_{0}}=\frac{\mu(t)}{1+\mu(t)}, \quad \mu(t)=\frac{\rho(t)}{\rho_{0}}-1
$$

Here, from Bragg's diffraction law applied at initial moment of irradiation, the Bragg angle is $\theta(t=0)=\theta_{0}$, and after irradiation time $t$ is $\theta(t)=\theta_{0}+\Delta \theta(t), \rho_{0}$ is initial density of the sample, $\rho(t)$ is density after irradiation time $t$

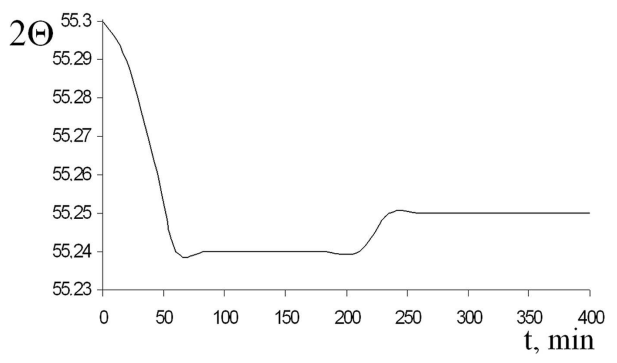

Fig. 3. Twofold Bragg diffraction angle $2 \Theta^{\circ}$ dependence on time measured using $\mathrm{Cu}$ anode $\mathrm{X}$-ray characteristic spectral line $K_{\alpha_{1}}$ of wavelength $\lambda=1.5405 \AA$ for anode voltage $U_{\mathrm{a}}=20 \mathrm{kV}$ and tube current $I_{\mathrm{a}}=20 \mathrm{~mA}$.

Using measured Bragg diffraction angle dependence on time and Eq. (4), we obtain fractional lattice spacing decreasing between the crystallographic planes (202) $\Delta d / d=0.001$ after $60 \mathrm{~min}$ irradiation. In this process we have production of vacancies and point defects. Later for long time intervals $(150 \mathrm{~min})$ we have some constant fractional spacing meanings. For time interval from 70 min till $220 \mathrm{~min}$ we have saturation of sample by diffusing vacancies. Later some processes occur without changes spacing in lattice by irradiated chemical reactions.

\section{Infrared investigations}

Information from diffraction spectra of X-rays [2] and electrical measurements [4] is not sufficient. We obtained additional information performing infrared measurements of alpha quartz, after $24 \mathrm{~h}$ irradiation with dose $6.37 \times 10^{6}$ Gy of X-rays from diffractometer DPOH$-3 \mathrm{M}(\mathrm{Cu}$ anode voltage $30 \mathrm{kV}$ and tube current $12 \mathrm{~mA}$ ). We can use soft X-rays for creation and destruction of defects in quartz, instead high gamma ray doses [12], soft X-rays can also generate vacancies in quartz like in crystal silicon [1].

Infrared measurements for investigation of modified point defects were performed with Vertex 70 Fourier interferometer manufactured by Bruckner. The detector system was cooled to liquid nitrogen temperature, spectral range was chosen from 500 to $5000 \mathrm{~cm}^{-1}$ with $1 \mathrm{~cm}^{-1}$ step and signal average of 128 scans. Sample was mounted in a special optical two-mirror system, which was placed between detector and interferometer output; this way only reflected signal from the sample surface was recorded. All measurements were taken at room temperature in closed chamber with dried air. Results are presented in Figs. 4 and Fig. 5.

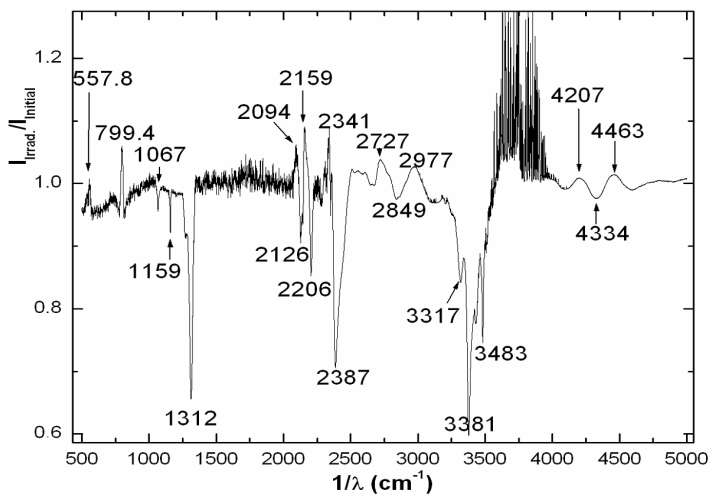

Fig. 4. Dependence of relation reflections $I_{\text {irrad }} / I_{\text {initial }}$ of irradiated and non-irradiated sides of alpha quartz on $1 / \lambda$.

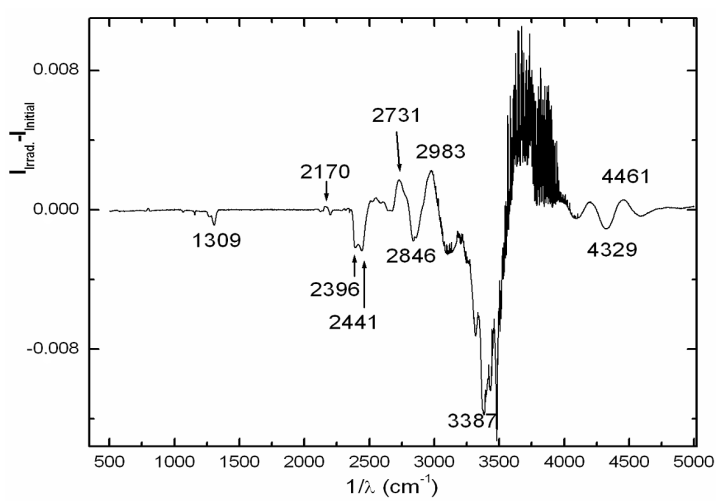

Fig. 5. Dependence of relative intensities differences $I_{\text {irrad }}-I_{\text {initial }}$ of reflections from irradiated and non-irradiated side of alpha quartz on $1 / \lambda$.

Infrared absorption spectrum of irradiated quartz presented as $I_{\text {irrad }} / I_{\text {initial }}$ is shown in Fig. 4 . In such presentation spectral bands pointing down are new band which appeared in quartz sample after the irradiation, while the bands pointing up $\left(557.8 \mathrm{~cm}^{-1}\right.$ and $\left.799.4 \mathrm{~cm}^{-1}\right)$ can be considered as quartz bands which vanish after the irradiation. Some shifts of quartz infrared absorption bands are also observed as changes of widths of bands after the irradiation. For instance, the band at $2094 \mathrm{~cm}^{-1}$ shifts to $2126 \mathrm{~cm}^{-1}$ and band at $2159 \mathrm{~cm}^{-1}$ shifts to $2206 \mathrm{~cm}^{-1}$.

Irradiation creates $\mathrm{Al}-\mathrm{OH}$ (band $3381 \mathrm{~cm}^{-1}$ presented in Fig. 4) and $\mathrm{Al}-\mathrm{h}$ centers [13] by replacing alkali ions by protons and holes. In action of Auger's effects we have similar changes in lattice as exposed to gamma rays [3]. During irradiation trivalent $\mathrm{Al}$ ions transform to tetravalent and can be swept by the Coulomb interaction into interstitial position creating metastable vacancies [12]. 
When the irradiation is interrupted, there can be created a population of $\left[\mathrm{Al}_{\mathrm{Si}} \mathrm{O}_{4}\right]^{-},\left[\mathrm{Al}_{\mathrm{Si}} \mathrm{O}_{4} / \mathrm{h}^{+}\right]^{0}, \mathrm{M}^{+}, \mathrm{h}^{+}$, $\mathrm{e}^{-}$and $\mathrm{M}_{i}[14]$. Here $\mathrm{M}$ are alkali metals. We obtained $\left[\mathrm{Al}_{\mathrm{Si}} \mathrm{O}_{4} / \mathrm{h}^{+}\right]^{0}$ centerscausing the absorption band [14] in visible region after irradiation, because irradiated side of our sample became "smoky" (darker).

During irradiation the following reactions play important role [14]:

$$
\begin{aligned}
& {\left[\mathrm{Al}_{\mathrm{Si}} \mathrm{O}_{4} / \mathrm{M}^{+}\right]+\gamma \rightarrow\left[\mathrm{Al}_{\mathrm{Si}} \mathrm{O}_{4} / \mathrm{h}^{+}\right]^{0}+\mathrm{M}^{+}+\mathrm{e}^{-},} \\
& \mathrm{M}_{i}+\gamma \rightarrow \mathrm{M}^{+}+\mathrm{e}^{-} .
\end{aligned}
$$

New growing band $3486 \mathrm{~cm}^{-1}$ in Fig. 4 can be assigned as Li dependent [14]. Lithium should give the best condition for color formation in irradiated quartz after heat treatment. This result differs from $[14,15]$ where there was observed the reduction of Li-dependent band in the natural quartz after irradiation with $\gamma$ rays at room temperature.

After irradiation we measured change of band $\left(3380 \mathrm{~cm}^{-1}\right)$ for $\mathrm{Al}-\mathrm{OH}$ center [14] whose shifted band is $3387 \mathrm{~cm}^{-1}$ and is presented in Fig. 5, for generated $\mathrm{Al}-\mathrm{O}$ center and negative vacancies. Diffusion of generated negative vacancies eliminates centers $(\mathrm{Al}-\mathrm{h})$ and together with reactions (7) initiates decreasing conductivity. In the region of $3655 \mathrm{~cm}^{-1}$ band we have modified point defects. Broader $3620-3680 \mathrm{~cm}^{-1}$ band in Figs. 4 and 5 represent [13] $\mathrm{H}_{2} \mathrm{O}$ absorption centers, whose vanishing can be explained as destruction of $\mathrm{O}-\mathrm{H}$ groups by irradiation [16]. Broadening of this band can be explained as absorption of $\mathrm{H}_{2} \mathrm{O}$ on the samples surface.

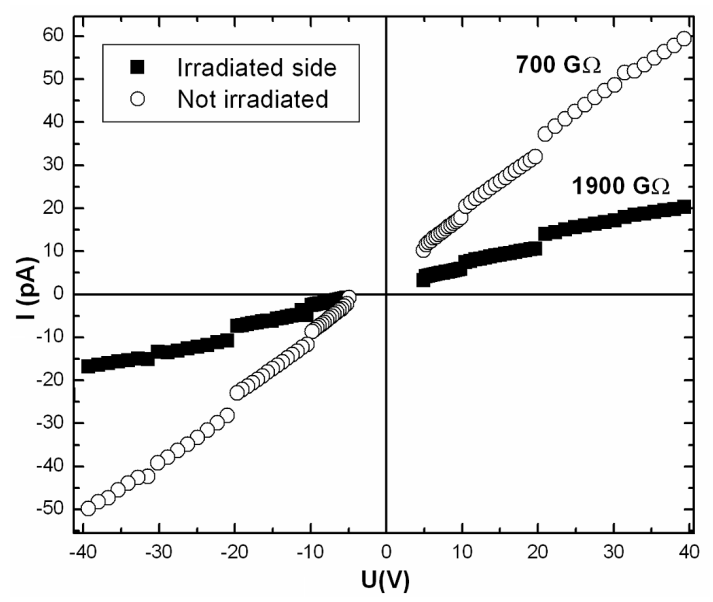

Fig. 6. Current-voltage characteristics of irradiated and non-irradiated sides of $2 \mathrm{~mm}$ thickness alpha quartz sample after $24 \mathrm{~h}$ of irradiation. Irradiation was performed with diffractometer DPOH-3M (Russian device) for anode voltage $30 \mathrm{kV}$ and X-rays tube current $12 \mathrm{~mA}$. At low currents and voltages $I-V$ characteristics do not cross zero point because of sample polarization. The resistivity of irradiated side has become $\approx 3$ times greater than the other side. $I-V$ measurements were performed with Keithley 6430 multimeter at room temperature in dark.
Changes of conductivity of alpha quartz after the same irradiation were measured. Sample of quartz plate was irradiated from one side. The opposite side of $2 \mathrm{~mm}$ thickness sample remained unchanged because of limited X-ray penetration depth. Two pairs of electrical contact islands of similar size and configuration at both sides of sample were formed with silver paste and connected with cooper wires. We measured separately $I-V$ characteristics for each side of the sample in few min time interval, $24 \mathrm{~h}$ after irradiation. They showed near to ohmic behavior (Fig. 6) as the consequence of sample high resistivity and completely depleted space charge region [17].

It is interesting to remark that irradiation of synthetic quartz crystals by X-rays over a period of several h enhances electrical conductivity [18]. This enhanced conductivity is produced by migration (activation energy for migration is $0.14 \mathrm{eV}$ ) of $\mathrm{M}^{+}$alkali ions along $c$-axis channels which are liberated during the irradiation. Presence of shallow traps $[18]$ for free $\mathrm{M}^{+}$alkali ions in natural crystals essentially changes conductivity after irradiation.

\section{Summary}

The results presented in this paper strongly depend on the presence of impurities in the quartz. We produced the vacancies by irradiation with soft X-rays as a result of presence of $\mathrm{Si}, \mathrm{Al}$, and alkali ions [3] in the quartz lattice.

From diffraction measurements and calculated root of mean square displacements of atoms in lattice, we cannot understand what point defects were swept and created in lattice for processes presented in Figs. 1 and 2 for beginning of irradiation. The irradiation has influence on distances between planes (202), density and strain of sample. The results presented in Figs. 2 and 3 for time interval from 50 min to 300 min show that processes, when number of point defects and displacements must increase, have weak connection with distances between planes (202). The oscillations of diffraction peak intensities presented in Fig. 1 can be connected with generated metastable vacancies and lattice relaxations [5]. These relaxations can be generated by replacing in lattice $\mathrm{Si}^{4+}$ ion by $\mathrm{Al}^{3+}$ ion and charge compensators $\left(\mathrm{Li}^{+}, \mathrm{Na}^{+}\right.$, $\mathrm{H}^{+}$). These defects form electric dipoles, which have six equivalent orientations [19]. The transitions between them require the migration or jumps of $\mathrm{Al}^{3+}$ ion. These electric dipoles interacting with lattice are the cause of dielectric constant [1] and quartz lattice relaxations.

From made infrared measurements, we did not verify formation of centers $\left(5640 \mathrm{~cm}^{-1}\right.$ band) after irradiation, but our alpha quartz became smoky. We can assert that we obtained $\left[\mathrm{Al}_{\mathrm{Si}} \mathrm{O}_{4} / \mathrm{h}^{+}\right]^{0}$ centers causing the absorption band [14] in visible region. From infrared measurements presented in Figs. 4 and 5 we obtained some vanishing 3620-3680 $\mathrm{cm}^{-1}$ absorption band representing the $\mathrm{H}_{2} \mathrm{O}$ centers. We used natural quartz which is intersected by microscopic channels filled by water [20]. These vanishing absorption bands can be explained as coming from destruction of $\mathrm{O}-\mathrm{H}$ groups by irradiation [16]. 


\section{References}

[1] A.J. Janavičius, J. Banys, R. Purlys, S. Balakauskas, Lith. J. Phys. 42, 337 (2002).

[2] V.P. Klad'ko, D.O. Grigoriev, L.I. Datsenko, V.F. Machulin, I.V. Prokopenko, Semicond. Phys., Quant. Electron. Optoelectron. 2, 157 (1999).

[3] V.S. Vavilov, A.E. Kiv, O.R. Nijazova, Mechanism of Producing and Migration of Defects in Semiconductors, Science, Moscow 1981, p. 368 (in Russian).

[4] R. Purlys, A.J. Janavičius, A. Mekys, S. Balakauskas, J. Storasta, Lith. J. Phys. 41, 376 (2001).

[5] A.J. Janavičius, Ž. Norgèla, R. Purlys, Acta Phys. Pol. A 104, 459 (2003).

[6] A.J. Janavičius, Ž. Norgèla, R. Purlys, Eur. J. Appl. Phys. 29, 127 (2005).

[7] A.J. Janavičius, J. Storasta, R. Purlys, A. Mekys, S. Balakauskas, Ž. Norgèla, Acta Phys. Pol. A $\mathbf{1 1 2}$, 55 (2007).

[8] J. Bourgoin, M. Lannoo, Point Defects in Semiconductors II, Experimental Aspects, Springer-Verlag, Berlin 1983, p. 305.

[9] G.D. Watkins, Mater. Sci. Semicond. Proc. 3, 227 (2000).
[10] S. Takagi, J. Phys. Soc. Jap. 26, 1239 (1969).

[11] S.J. Turneaure, Y.M. Gupta, Appl. Phys. Lett. 90 , 051905 (2007).

[12] G.F. Savakuchi, E. Okuno, Nuclear Instrum. Methods Phys. Res. B 218, 217 (2004).

[13] H.G. Lipson, IEEE Trans. Ultrason. Ferroelectr. Freq. Control 37, 524 (1990).

[14] E.H. Martins Nunes, F. Soares Lameiras, Mater. Res. 8, No. 3, Săo Carlos July/Sept. (2005).

[15] H. Bahadur, J. Appl. Phys. 66, 4973 (1989).

[16] L. Skuja, B. Guttler, D. Schiel, A.R. Silin, Phys. Rev. B 58, 14296 (1998).

[17] B.K. Jones, J. Santana, M. McPherson, Solid State Commun. 105, 547 (1998)

[18] H. Jain, A.S. Nowick, J. Appl. Phys. 53, 485 (1982).

[19] J. Toulouse, S. Ling, A.S. Nowick, Phys. Rev. B 37, 7070 (1988).

[20] G.A. Yurgenson, V.D. Perevertaev, N.I. Dubovikov, Zh. Prikl. Spektrosk. 25, 1045 (1975) (in Russian). 